\title{
Zoning based MAC with support for recharging process in WSN
}

\author{
Mohammad Shahnoor Islam Khan, Jelena Mišić and Vojislav B. Mišić \\ Ryerson University, Toronto, ON, Canada M5B 2K3
}

\begin{abstract}
Radio-frequency (RF) based recharging of sensor nodes is a promising way to reduce maintenance and extend the operational life of wireless sensor networks. However RF attenuation causes the network nodes with largest distance from the access point (master node) to dictate the rate of recharging which imposes unnecessary breaks in the operation of nodes closer to the master. This deteriorates the throughput of the nodes close to the master. To solve this problem we have designed location aided MAC protocol which supports recharging such that all nodes deplete their batteries at approximately the same time so that recharging pulse comes on time for all the nodes. To achieve that we have partitioned network nodes into circular zones around the master and assigned implicit priorities among the zones. Priorities decrease towards the edge of the network and regulate relative throughputs among the zones. We have built probabilistic performance model to evaluate the impact of the recharging process on data communication of different zones by varying traffic load and network size.
\end{abstract}

\section{INTRODUCTION}

Periodic recharging of sensor nodes is a promising approach as it reduces maintenance and extend the functional life of wireless sensor networks (WSNs) [2]. WSNs can use energy from the environment referred as 'energy harvesting' [4], or recharging can be accomplished via high energy pulses from the access point (known as master node, coordinator or base station) [12]. The former technique has advantage over energy harvesting as it does not require to depend on the ambient condition of nature. Due to this, as well as on account of its higher controllability, RF recharging is getting more acceptance in recent years [14], [13].

RF recharging may use separate frequency band from regular data communications, or the two can share the same RF band. The former approach requires two antenna systems and two RF transceivers [12] and it can guarantee uninterrupted data communication [11]. Still, the use of a single RF band is interesting on account of hardware simplicity and less usage of frequency resource. But it has an adverse impact on data throughput, as both data communication and recharging can not be done simultaneously [9]. Careful tailoring of the protocol and detailed analysis is needed to minimize performance implications of the interplay between recharging and data communications.

One of the challenging issues of single band RF recharging is to minimize number of recharging events. Single band RF technique is mainly influenced by the farthest node [5]. Due to the minimum energy gain, the node's energy dries up sooner and it asks for recharging earlier compared to the other nodes. Other nodes can not proceed with normal data communication although they have sufficient energy. To address this critical problem, we have divided the network in circular zones around the coordinator. Zones are assigned numbers $1,2,3 \ldots n_{z}$ according to their distance from the coordinator with highest priority assigned to zone 1 which is closest to the coordinator. Zone priority is further translated to the frequency of polling in the MAC protocol. Zoning approach attempts to equalize periods between recharging for all zones on behalf of different data throughputs among the zones. This can be beneficial for the networks with heterogeneous traffic and controllable locations of the nodes.

General treatment of energy replenishment including battery replacement or conventional recharging was presented in [7]. A MAC protocol that explicitly requests energy replenishment through a subsequent RF pulse has been studied in [12]. To enhance battery lifetime, clustering (zoning) of sensor nodes have been implemented by segmenting local and global traffics [6]. But it does not discuss on how to improve the energy consumption of nodes when local master node communicates with different distant nodes.

In this paper, we propose a simple MAC protocol inspired by Bluetooth [10] in which ordinary nodes in different zones are polled with varying number of times by the master node and in-band recharging is performed when explicitly requested by a sensor node. The polling is done in round robin fashion and a node's number of packet transmissions per cycle depends on the assigned zone. We then describe a probabilistic model that allows us to evaluate the performance of data communications in this setup, in particular the interplay between recharging and data communications.

The paper is organized as follows: zoning principle is explained in Section II. MAC protocol for nodes in different zones along with the RF recharging process, is described in Section III. Probability distribution of periods between successive recharging events are discussed in Section IV. In Section V we model vacation period of a node. Performance of the proposed MAC protocol is analysed in Section VI. Section VII concludes the paper and highlights some promising avenues for future research.

\section{ZONING DESCRIPTION}

We assume that the network consists of an access point or master node that has an inexhaustible power supply and a total of $m$ sensor nodes that can be recharged through RF pulses. The sensor nodes are scattered in a circular area with the master node located at the center shown in Fig. 1. 


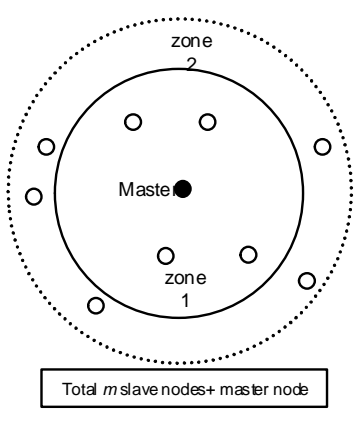

Fig. 1. Logical presentation of the network with zones.

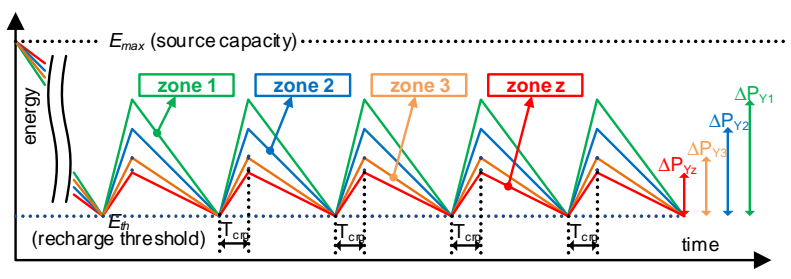

Fig. 2. Node recharging and energy expenditure cycles

Each of the nodes senses the environmental variable of interest and attempts to send it to the master when polled. Each sensing result can fit in a single packet; sensing is performed at random times that follow a Poisson process with an arrival rate of $\lambda$.

Packet transmission can be corrupted by noise and interference (at an error rate of $\epsilon_{p} \ll 1$ per packet), which is why the coordinator will acknowledge successfully received packets. To keep communications to a minimum, delayed acknowledgement is used: namely, the next POLL packet destined to the same node will have its ACK bit set if the data packet was successfully received. Packet retransmission will be attempted up to $n_{a}$ times; to this end, the node must retain a packet just transmitted until the acknowledgement is received or the retry limit is reached.

All nodes start with identical energy reserve $E_{\text {max }}$. During normal network operation, energy is depleted in various node activities. When the energy level of the node drops below a predefined threshold $E_{t h r}$, the node will send its DATA or NULL packet with the HELP bit set. Upon learning that

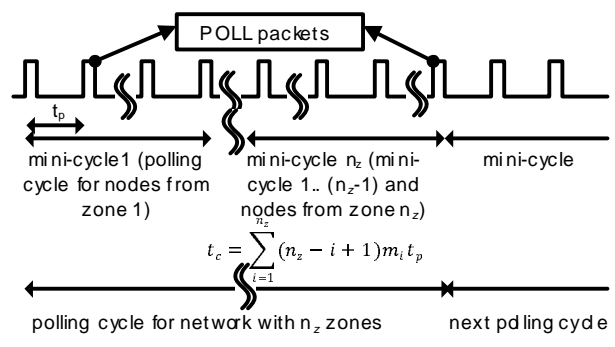

(a) Network with $n_{z}$ zones.

Fig. 3. Polling cycle is constructed by nesting partial-cycles. recharge is needed, the master node will send a recharge pulse on the same RF frequency/band used for data communications, thus temporarily disrupting regular communications. To benefit from the unscheduled recharge, each node keeps its RF recharging circuitry active (i.e., in the receiving mode) between successive polls. The energy increment received by a node $k=1 . . m$ through recharge can be calculated using the Friis equation [3]

$$
\Delta E_{k}=t_{r} P_{r} \eta G_{r} G_{t}\left(\frac{\lambda_{w}}{4 \pi d_{k}}\right)^{\gamma}
$$

where $t_{r}$ and $P_{r}$ denote duration and power of the recharge pulse, respectively; $\eta$ is the coefficient of efficiency of RF power conversion; $G_{r}$ and $G_{t}$ are the antenna gains at the receiver and transmitter, respectively; $\lambda_{w}$ is the wavelength, $d_{k} \gg \lambda_{w}$ is the distance between the transmitter and the receiver (i.e., the coordinator and the node in question), and $\gamma$ is the path loss coefficient 2 in free space, but as high as 4 or 5 in closed spaces. General process of a node's energy consumption due to transmit data and gain in energy through recharging is shown in Fig. 2.

\section{ZONING WITHIN MAC}

In zoning approach, sensor field is partitioned into $n_{z}>1$ circular zones or coronas. The nodes are assigned to zones based on their distance from the master node. Difference in recharging energy gains for nodes in two consecutive zones $i$ and $(i+1)$ is smaller than a predefined value $\sigma \leq\left(\Delta E^{i}-\Delta E^{i+1}\right)$. Example of the network with two zones is shown in Fig. 1, where zones 1 and 2 have $m_{1}$ and $m_{2}$ nodes respectively.

The basis of the MAC protocol is polling where master polls each node using polling packet in order to obtain single data packet. If node's buffer is empty it will reply with null packet. Polling cycle $t_{c}$ is the period where each network node is polled at least once and in our case it will be determined by polls to the node furthest from the master. In order to implement zoning priorities polling cycle is divided into partial-cycles which are given indices $1,2, \ldots n_{z}$. In partial-cycle 1 only nodes in first zone are polled for a single packet from each. In the partial-cycle $i$ nodes from zones 1 , 2 up to $i$ are polled, and so on. Finally, in the partial-cycle $n_{z}$ nodes from all zones are polled. Complete polling cycle consists of all $n_{z}$ partial cycles. Therefore the effect of this implicit distance based prioritizing is that nodes from zone $i$, $i=1,2, \ldots n_{z}$ can transmit $\left(n_{z}-i+1\right)$ packets in the polling cycle. Assuming that zone $i$, has $m_{i}$ nodes, total number of data packets sent by all nodes in that zone is $m_{i}\left(n_{z}-i+1\right)$ and total number of packets transmitted in the cycle will be $N_{d}=\sum_{i=1}^{n_{z}} m_{i}\left(n_{z}-i+1\right)$.

Note that, if any node sends recharging requests any time of the polling cycle, the master node broadcasts a special POLL message informing about the pending recharging pulse. Normal operation of the cycle is paused and all the nodes are ready for being recharged. 
TABLE I

ENERGY CONSUMPTION COMPONENTS OF A SESNING NODE.

\begin{tabular}{ll}
\hline Basic tasks & \\
\hline sensing a DATA packet & $E_{s}$ \\
listening a POLL packet & $E_{p}$ \\
listening to header part of a packet & $E_{h}$ \\
transmitting a DATA packet & $E_{d}$ \\
transmitting a NULL packet & $E_{d 0}$ \\
listening for a recharge packet & $E_{r}$ \\
\hline Energy budget to send single packet from zone $i$ & \\
\hline NULL packet $E_{n}^{i}=E_{p o l l}+\frac{\left(n_{c}(z)-1\right)}{z-i+1} E_{h}+E_{d 0}$ & \\
DATA packet $E_{t}^{i}=E_{s}+E_{p o l l}+\frac{\left(n_{c}(z)-1\right)}{z-i+1} E_{h}+E_{d}$ & \\
DATA packet retransmission $E_{r t}^{i}=E_{p o l l}+\frac{\left(n_{c}(z)-1\right)}{z-i+1} E_{h}+E_{d}$ &
\end{tabular}

In order to calculate the mean duration of total polling cycle we will assume that polling packet consumes 1 time unit while data packet consumes $f$ time units. Polling packets are sent after every $t_{p}=(1+f)$ time units. If nodes always have data packets to transmit (in saturation condition) polling cycle $t_{c}$ can be calculated as

$$
t_{c}=\sum_{i=1}^{n_{z}} m_{i}\left(n_{z}-i+1\right) t_{p}
$$

In order to calculate energy consumption we need to take into account all atomic activities of sensing node which include sensing, transmission/re-transmission, listening and recharging as shown in Table I. If we assume that the energy needed of sensing the actual data value can be lumped together with the energy needed to send the resulting packet, and that the energy consumed during the idle period between polls is negligible, mean energy expenditure in a cycle $t_{c}$ by a node of zone $i$ can be calculated using the energy expenditure of atomic activities listed in Table I as:

$$
\begin{aligned}
\overline{E_{c}^{i}} & \left.\approx N_{d}-\left(n_{z}-i+1\right)\right) E_{h}+\left(n_{z}-i+1\right)\left(E_{p}+E_{d}\right) \\
& +\left(n_{z}-i+1\right)\left(1-\epsilon_{p}\right) E_{s}, \text { for } 1 \leq i \leq n_{z}
\end{aligned}
$$

Total energy consumed to transmit $N_{d}$ in a complete polling cycle $t_{c}$ can be calculated as

$$
E_{\text {Total }}=\sum_{i=1}^{n_{z}} m_{i} \overline{E_{c}^{i}}
$$

\section{A. Throughput Analysis: Zoned vs. Non-zoned Network}

During recharging transmission is not possible. Therefore, if recharging is frequently triggered by the furthermost node, other nodes which still have energy resources will be unnecessarily prevented from transmission and their traffic backlog will build up. In order to alleviate this problem, our scheme allows higher utilization of the nodes closer to the master.

Fig. 4 explains how differentiated data rates of nodes according to zone priority increase the network data throughput. Let us assume that four nodes $i=1,2,3,4$ are placed at $1 \mathrm{~m}, 1.41 \mathrm{~m} 1.73 \mathrm{~m}$ and $2 \mathrm{~m}$ away from the master node. Recharging duration is set to 100 time units. Period between two recharging events (also known as operational period) would be 100, 200, 300 and 400 cycles for nodes located in the descending order of distances shown in Fig. 4(a). Node utilization is then $50 \%, 66.67 \%, 75 \%$ and $80 \%$. When all these four nodes are functioning together, furthest node will ask for recharging after each 100 operational cycles. In that case, utilization of all nodes will drop down to $50 \%$ as operational periods of closer nodes are interrupted by the recharging call of the farthest node. Without zoning, each node has equal data processing rate. If data transmission rate is $x$ packets per 100 time units, the network can process $4 x$ packets every 500 time units (400 time units that follows round robin scheduling for four nodes plus 100 unit recharging duration). Data throughput for the network will be $(4 x / 500)$ packets per time unit.

Fig. 4(b) explains recharging event and operational period after implementing zoning (i.e. different data processing rate). In this scenario, node $i$ can send up to $5-i$ packets during a single polling cycle according to the descending order of distances from the master node and the polling cycle length will be $1+2+3+4=10$ packet times. The farthest node will get single opportunity to send data in every 10 polls instead of single chance in every 4 polls without zoning. However, as the nodes send different numbers of packets, their energy consumption rate will be different.

After implementation of zoning the operation period will be extended to 1000 time units and recharging pulse lasts 100 units. Then nodes in zones $1,2,3$, and 4 will be able to send $x, 2 x, 3 x$ and $4 x$ packets respectively during that time. The network can send $(x+2 x+3 x+4 x)=10 x$ packets in 1100 time units. Network throughput will be $x / 110$ packets per time unit which renders throughput increase of $12 \%$. If the recharging period is changed to 1000 units keeping all other parameters unchanged the throughput improvement would be around $43 \%$.

\section{RECHARGING PERIOD}

The time duration between two successive recharging pulses is a random variable due to the random packet loss and different energy consumption rate for different packet types and quantities. We need to focus on the energy expenditure of a node which can be calculated by measuring total number of polling cycles spent between two consecutive recharging events. This may be fulfilled by measuring the joint probability distribution of the number of polling cycles and energy consumed in each polling cycle.

Let $x$ be the variable for sensing energy unit of a single packet, $y$ be energy required to transmit a packet and $t$ denote the variable to present required time. Probability generating function (PGF) of required energy and time to send a DATA packet successfully can be expressed as

$$
E_{s u b}(x, y, t)=\frac{x y t \sum_{k=0}^{n_{a}}(y t)^{k} \epsilon_{p}^{k}}{\sum_{k=0}^{n_{a}} \epsilon_{p}^{k}}
$$

A node in a zone $i$ sends NULL packet when it does not have any data with $\left(1-\rho_{t o t}^{i}\right)$ probability where $\rho_{t o t}^{i}$ is the 


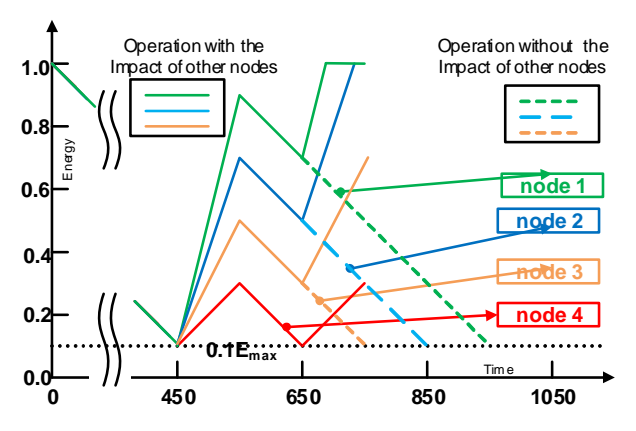

(a) Impact of early recharging call initiated by the farthest node on other nodes operation.

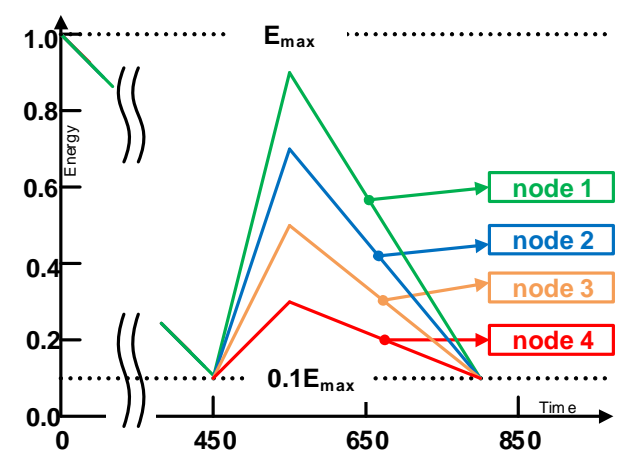

(b) Operational duration of nodes after applying zoning.

Fig. 4. Impact zoning on network throughput

effective offered load of the node. This value varies for nodes in different zones. Let $\phi$ be the variable for required energy unit to send a NULL packet. PGF of required energy and time to send a packet (DATA/NULL) successfully is

$$
\begin{aligned}
E_{\text {full }}(i, x, y, t, \phi)= & \rho_{\text {tot }}^{i} E_{\text {sub }}(x, y, t) \\
& +\left[1-\rho_{\text {tot }}^{i}\right] \phi t
\end{aligned}
$$

A node in zone $i$ can send $\left(n_{z}-i+1\right)$ number of packets. PGF for successful transmitting this number of packets will be $\left(E_{\text {full }}(i, x, y, t, \phi)\right)^{n_{z}-i+1}$.

A node in the zone keeps sending packets until its energy level goes below a threshold value $E_{t h r}^{i}$. Practically, this threshold value should be zone specific and $E_{t h r}^{i}>E_{t h r}^{i+1}$ (closer zone requires more threshold value as it sends more packets in a polling cycle). $E_{b} i=E_{t h r}^{i}+\Delta E_{i}$ is the energy budget of a node located in $i^{\text {th }}$ zone.

Between successive recharging points, the node can complete polling cycles range between $n_{\min }=\frac{\Delta E_{i}}{\left(n_{z}-i+1\right)\left[\left(n_{a}+1\right) E_{r t}^{i}+E_{s}\right]}$ and $n_{\max }=\frac{\Delta E_{i}}{\left(n_{z}-i+1\right) E_{n}^{i}}$.

PGF for all possible number of packet transmissions when energy resource can be exhausted is

$$
S E_{\text {full }}(i, x, y, t, \phi)=\frac{\sum_{k=n_{\min }}^{n_{\max }} E_{f u l l}(i, x, y, t, \phi)^{\left(n_{z}-i+1\right) k}}{n_{\max }-n_{\min }+1}
$$

We can merge all the energy units $x, y$ and $\phi$ to single energy units into a new single energy unit $v$ by applying the transformation algorithm in [9]. Unit value of new energy variable is equivalent to the unit value of sensing energy variable $x$.

$$
S E_{f u l l}(i, x, y, t, \phi) \stackrel{\text { Transformation }}{\longrightarrow} S E_{\text {full }}(i, v, t)
$$

Let $\operatorname{cov}^{i}(j)$ be coefficient of $v^{j}$ in $S E_{f u l l}(i, v, t)$ for a node in zone $i . \max _{v}^{i}$ be the maximum exponent value on $v$ in $S E_{\text {full }}(i, v, t)$.

Partial PGF of energy consumption that exceeds energy budget $\Delta E_{b}^{i}$ can be expressed as

$$
T_{o}^{i}(t)=\sum_{j=E_{n}^{i}}^{\max _{v}^{i}} \operatorname{cov}^{i}(j) \text { where } E_{n}^{i}=\frac{E_{b}^{i}}{E_{d 0}}
$$

Complete PGF for time units that consumes more than energy budget is expressed as

$$
T_{\text {out }}^{i}(t)=\frac{T_{o}^{i}(t)}{T_{o}^{i}(1)}
$$

Mean duration between two consecutive recharging events for a node in zone $i$ is represented as $\left.T^{i^{\prime}}(t)\right|_{t=1}$. Energy outage probability $p_{\text {out }}^{i}=\frac{1}{\left.T^{i^{\prime}}(t)\right|_{t=1}}$ of the node is simply the reciprocal of mean duration.

\section{VACATION ANALYSIS}

Vacation is a period when node is not able to transmit. It is composed of recharging and activities of other nodes due to round robin polling. A node in $i$-th zone sends $\left(n_{z}-i+1\right)$ packets in the uplink and receives same number of POLL packets in the downlink. PGFs for uplink data transmission and downlink POLLs will be $G_{p}^{i}(z)=z^{n_{z}-i+1}$ and $G_{d}^{i}(z)=$ $z^{n_{z}-i+1}$ respectively. Mean duration to send this number packets and to receive POLLs will be $\left(G_{p}^{i^{\prime}}(z)+G_{d}^{i^{\prime}}(z)\right)$. Packet service rate is $\mu=\frac{n_{z}+i-1}{G_{p}^{i^{\prime}}(z)+G_{d}^{i^{\prime}}(z)}$. Offered load of a node in the $i^{\text {th }}$ zone will be $\rho^{i}=\frac{\lambda}{\mu}$

Presence of vacation $V(\bar{V}$ represents mean vacation time) increases the offered load to

$$
\rho_{\text {eff }}^{i}=\rho^{i}+\lambda \bar{V}
$$

Further, the use of transmission reliability effectively transforms the single packet transmission into a burst transmission with the PGF of

$$
G_{b}(z)=\frac{\sum_{k=0}^{n_{a}} z^{k} \epsilon_{p}^{k}}{\sum_{k=0}^{n_{a}} \epsilon_{p}^{k}}
$$

with mean value $\overline{G_{b}}=G_{b}^{\prime}(1)$. Therefore the total scaled uplink offered load becomes

$$
\rho_{\text {tot }}^{i}=\left(\rho^{i}+\lambda \bar{V}\right) \overline{G_{b}}
$$

Each node experiences two types of vacations.

- Cyclic vacation $V_{c}$ is due to the other nodes' active participation in sending packet to the master node. The 
PGF of this vacation is

$$
\begin{aligned}
V_{c y c}^{i}(z)= & \prod_{j=1}^{n_{z}}\left[\rho_{t o t}^{j} G_{p}^{j}(z) z^{n_{z}-j+1}\right. \\
& \left.-\left(1-\rho_{t o t}^{j}\right) z^{2\left(n_{z}-j+1\right)}\right]^{\alpha_{j}}
\end{aligned}
$$

where $\alpha_{j}=m_{j}$ if $i \neq j$; otherwise $\alpha_{j}=\left(m_{j}-1\right)$.

- Recharging vacation $V_{c r g}^{i}$ lasts for $T_{c r g}$ duration and the PGF of this vacation is

$$
V_{\text {crg }}^{i}(z)=p_{\text {out }}(i) z^{T_{\text {crg }}}+\left(1-p_{\text {out }}(i)\right)
$$

The probability distribution (expressed as a PGF) for the total vacation experienced by a single node is

$$
V_{i}(z)=V_{c y c}^{i}(z) V_{c r g}^{i}(z)
$$

Its mean value and standard deviation are calculated as

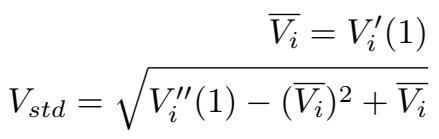

\section{PERFORMANCE ANALYSis}

We have implemented 3 zones in our experiment. Distance of the zone center to the master is set to $5 \mathrm{~m}, 7 \mathrm{~m}$ and $8.7 \mathrm{~m}$ for zones 1, 2 and 3 respectively. Each zone has equal number of nodes ranging from $m=3$ to 9 nodes. Nodes generate packets at a rate of $\lambda=0.00225 \cdots 0.00525$ with a stepwise increment value of 0.0005 packets per time unit. Packet error rate is assumed to be $\epsilon_{p}=0.02$. The packet retransmission limit was set to $n_{a}=3$. Both downlink (POLL) and uplink (DATA or NULL) packets last for one time slot. When requested, the coordinator sends a recharging pulse of power $0.1 \mathrm{~W}$ that lasts for 1000 slots. In our experiment, we have considered energy consumption values for different events, i.e., sensing packets, transmitting, according to the [1]. We have solved the system of equations described above using Maple 16 from Maplesoft, Inc. [8].

Our first set of experiments shows the main performance descriptors of network load and recharging as functions of packet generation rate $\lambda$ and network size $m$.

Figs. 5(a) to 5(c) show the total offered load mentioned in Eqn. (13). The offered load is approximately linearly related to the packet generation rate and network size. Zone 3 is having the highest offered load as it has the minimum processing time (A node in this zone can send 1 packet in a polling cycle $t_{c}$ ). On the other hand nodes in zone 1 have the lowest offered load.

Mean recharging period, expressed in polling cycles, is shown in Figs. 5(d) to 5(f). As can be seen, larger network size causes to faster battery depletion, since all nodes need to listen to all POLL packets. The packet arrival rate is small enough throughout the observed range, hence the probability of sending a NULL packet is very high when $m$ is smaller. As NULL packet consumes less energy compared to DATA packet, higher recharging period is observed for smaller network size $m$. Larger network size causes a node to have higher vacation period during which more packets arrive, hence the probability of the node's queue is empty gets reduced. Length of recharging periods for nodes in three different zones are similar which fulfils one of the important condition to maximize overall network throughput. This condition can not be met without the implementation of zoning.

The traffic rate $\lambda$ has strong impact on the length of recharging duration. Namely, higher traffic rate results in more data packets and fewer null packets, which causes a shorter recharging period. At the same time, higher traffic leads to more packet retransmissions which consumes energy besides regular packet transmissions. Recharging period is greatly reduced for larger network sizes, and it has less dependency on the traffic load. Outage probabilities in Figs. 5(g) to 5(i) are reciprocals of the mean durations between successive recharging points.

Descriptors of total vacation time are shown in Fig. 6. Mean vacation time increases with higher network size due to an increase in cyclic vacation. This period also increases slightly with higher traffic rate as it causes higher recharging period.

\section{CONCLUSION AND FUTURE WORK}

In this paper we have introduced prioritization in round robin MAC protocol with RF recharging. Priorities are assigned to nodes according to the distance from the master node. Nodes are grouped into circular areas around master called zones. Each zone is assigned priority such that nodes closest to the master have highest and nodes furthest from the master have lowest priority. Nodes in zones with higher priorities transmit more packets than low priority ones. The effect of this scheme is that nodes in different zones have different throughputs which balance out different levels of battery re-charging per zone. As a result nodes deplete their batteries in approximately same time. Therefore there is no unnecessary recharging of the nodes which takes away their communication time. Although zoning improves network throughput significantly, it does not guarantee fairness among the nodes. The farther nodes always get less opportunity to send data and packet delay of those nodes will be higher. However, zoning could be very good solution if network has heterogeneous nodes with different traffic intensity and node distances from the master can be set according to traffic.

\section{REFERENCES}

[1] B. Blum. Bluetooth low energy, A Very Low Power Solution. Technical report, Texas Instruments, Inc., Dallas, TX, 2012.

[2] Z. A. Eu, H.-P. Tan, and W. K. Seah. Design and performance analysis of MAC schemes for wireless sensor networks powered by ambient energy harvesting. Ad Hoc Networks, 9(3):300-323, May 2011.

[3] H. T. Friis. A note on a simple transmission formula. proc. IRE, 34(5):254-256, 1946.

[4] A. Kansal and M. B. Srivastava. An environmental energy harvesting framework for sensor networks. In Int. Symp. Low Power Electronics and Design (ISLPED 2003), pages 481-486, 2003.

[5] M. Khan, J. Mišić, and V. Mišić. Impact of network load on the performance of a polling mac with wireless recharging of nodes. IEEE Transactions on Emerging Topics in Computing, to appear.

[6] V. Kumar, S. Jain, S. Tiwari, et al. Energy efficient clustering algorithms in wireless sensor networks: A survey. IJCSI International Journal of Computer Science Issues, 8(5), 2011. 

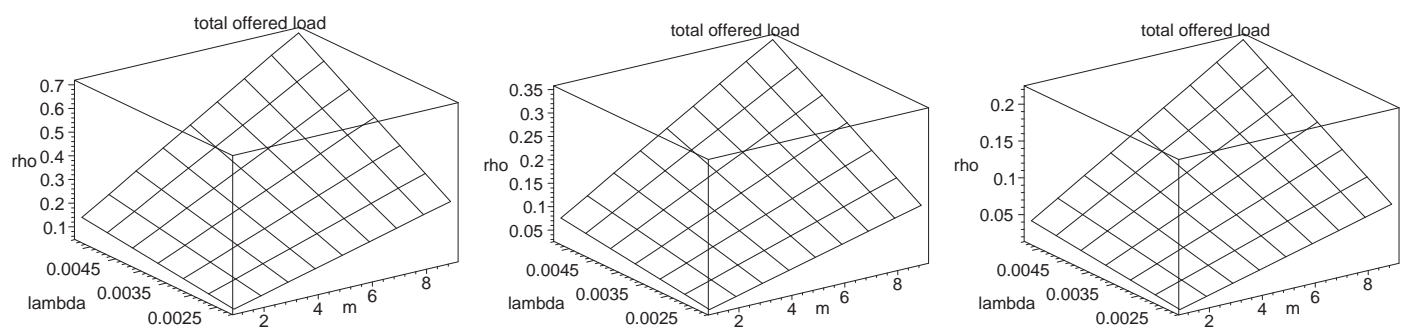

(a) Total offered load for a node zone 3. (b) Total offered load for a node zone 2. (c) Total offered load for a node zone 1.
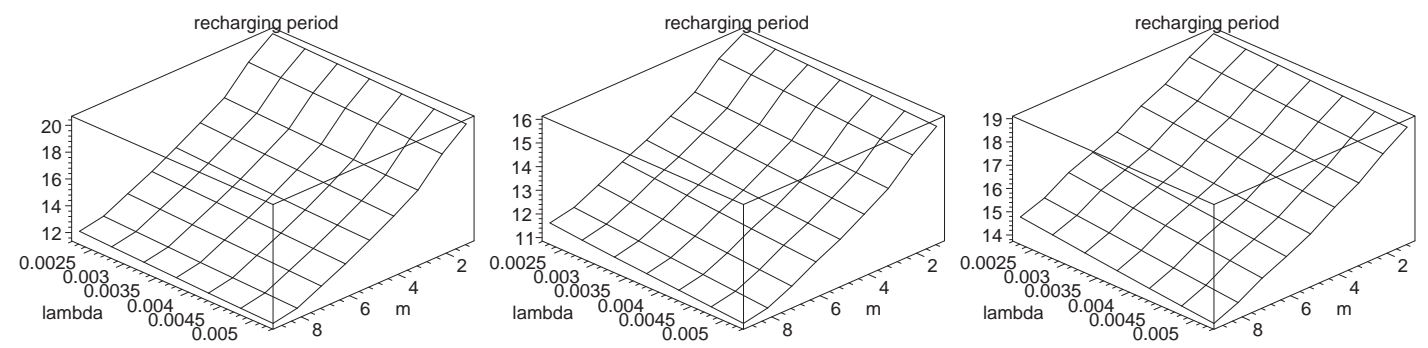

(d) Mean duration between two recharg- (e) Mean duration between two recharging events for nodes in zone 3 . ing events for nodes in zone 2 . ing events for nodes in zone 1.

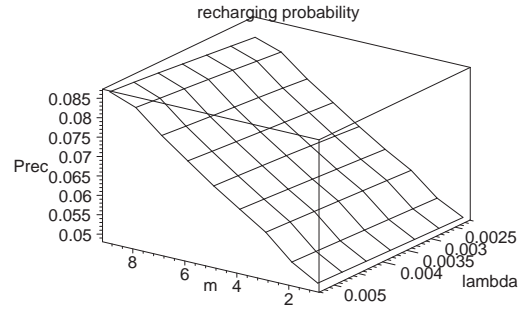

(g) Outage probability for a node zone 3 .

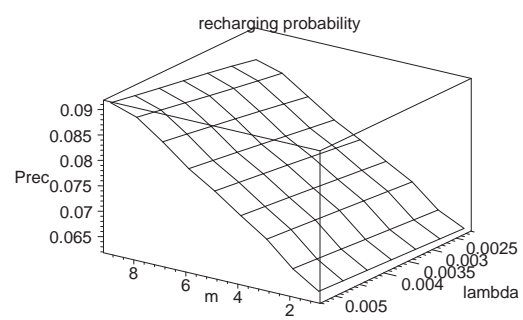

(h) Outage probability for a node zone 2 .

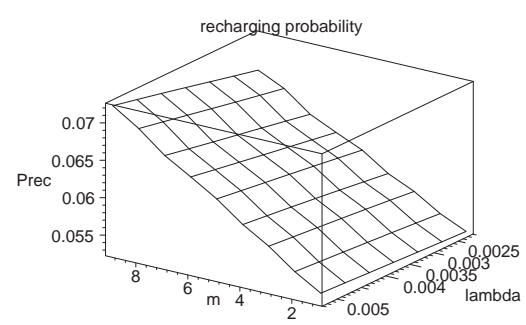

(i) Outage probability for a node zone 1 .

Fig. 5. Descriptors of load and recharging process (with zoning).

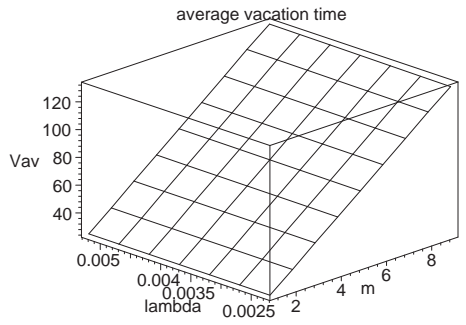

(a) Mean vacation for a node zone 3 .

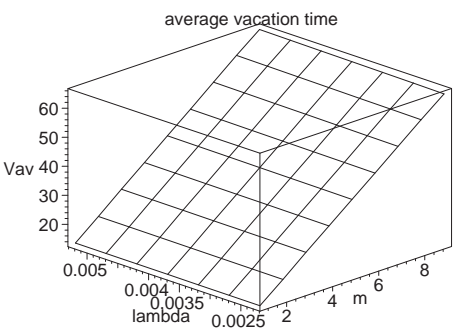

(b) Mean vacation for a node zone 2 .

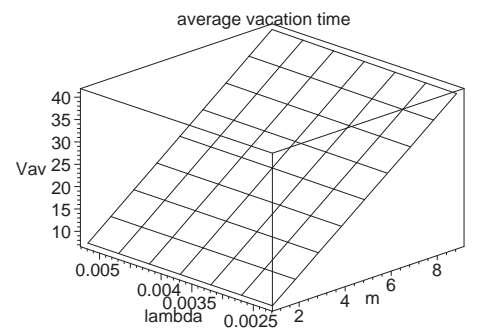

(c) Mean vacation for a node zone 1 .

Fig. 6. Vacation period (with zoning).

[7] J. Lei, R. Yates, and L. Greenstein. A generic model for optimizing single-hop transmission policy of replenishable sensors. IEEE Transactions on Wireless Communications, 8(2):547-551, 2009.

[8] Maplesoft, Inc. Maple 16. Waterloo, ON, Canada, 2013.

[9] J. Mišić, M. S. Khan, and V. B. Mišić. Performance of simple polling mac with wireless re-charging in the presence of noise. In Proceedings of the 17th ACM international conference on Modeling, analysis and simulation of wireless and mobile systems, pages 137-143. ACM, 2014.

[10] J. Mišić and V. B. Mišić. Performance Modeling and Analysis of Bluetooth Networks: Network Formation, Polling, Scheduling, and Traffic Control. CRC Press, Boca Raton, FL, July 2005.

[11] V. B. Mišić and J. Mišić. A polling MAC for wireless sensor networks with rf recharging of sensor nodes. In 27th Queen's Biennial Symposium on Communications, Kingston, ON, Canada, 2014.

[12] P. Nintanavongsa, M. Y. Naderi, and K. R. Chowdhury. Medium access control protocol design for sensors powered by wireless energy transfer. In IEEE INFOCOM, pages 150-154, Apr. 2013.

[13] L. Xie, Y. Shi, Y. T. Hou, and A. Lou. Wireless power transfer and applications to sensor networks. IEEE Wireless Communications, 20(4):140-145, 2013.

[14] L. Xie, Y. Shi, Y. T. Hou, and H. D. Sherali. Making sensor networks immortal: An energy-renewal approach with wireless power transfer. IEEE/ACM Transactions on Networking, 20(6):1748-1761, Dec. 2012. 